Rosângela Souza Lessa

(iD) https://orcid.org/0000-0002-6044-010X

Rita de Cássia Pereira Fernandes ${ }^{b}$

(iD) https://orcid.org/0000-0002-3353-5365

a Universidade Federal da Bahia (UFBA), Programa de Pós-Graduação em Saúde, Ambiente e Trabalho.

Salvador, BA, Brasil.

b Universidade Federal da Bahia (UFBA), Departamento de Medicina Preventiva e Social, Programa de Pós-Graduação em Saúde, Ambiente e Trabalho. Salvador, BA, Brasil.

Contato:

Rita de Cássia Pereira Fernandes

E-mail:

ritafernandes@ufba.br

Os autores declaram que o trabalho foi subvencionado por Bolsa de Mestrado fornecida pela Coordenação de Aperfeiçoamento de Pessoal de Nível Superior (Capes) e que não há conflitos de interesses.

As autoras informam que recortes do trabalho foram apresentados no $8^{\circ}$ Congresso Internacional de Fisioterapia, em 2016, e no $10^{\circ}$ Congresso Brasileiro de Epidemiologia, em 2017.

Trabalho baseado na dissertação de mestrado de Rosângela Souza Lessa intitulada "Dor em membros inferiores em trabalhadores da limpeza urbana", apresentada em 2015 no Programa de Pós-graduação Saúde, Ambiente e Trabalho da Universidade Federal da Bahia.
Recebido: 07/06/2019

Revisado: 09/01/2020

Aprovado: 10/06/2020

\section{Dor nas extremidades inferiores, demandas físicas e psicológicas em trabalhadores da limpeza urbana: estudo transversal}

\author{
Lower extremity pain, physical and psychological demands in \\ urban cleaning workers: a cross-sectional study
}

\section{Resumo}

Objetivo: descrever as características do trabalho e investigar a prevalência e os fatores associados à dor em membros inferiores em trabalhadores da limpeza urbana. Métodos: estudo de corte transversal, realizado na Bahia. Dados coletados entre 2009 e 2010. A dor foi avaliada por meio do Nordic Musculoskeletal Questionnaire, enquanto as demandas psicossociais no trabalho foram medidas pelo Job Content Questionnaire. Também foram avaliadas as demandas físicas, incluindo posturas gerais e manuseio de carga. A análise de regressão logística múltipla foi utilizada para identificar fatores associados à dor em membros inferiores. Resultados: 624 trabalhadores participaram da pesquisa. Constatou-se alta prevalência de dor em membros inferiores nos últimos sete dias $(23,7 \%)$ e nos últimos doze meses (42,1\%), sendo maior entre agentes de limpeza e coletores. A dor em membros inferiores foi associada a: mais de três anos de trabalho na empresa $(\mathrm{OR}=1,34)$; alta exposição ao manuseio de cargas $(\mathrm{OR}=1,35)$; demanda psicológica no trabalho $(\mathrm{OR}=1,87)$; e condicionamento físico insuficiente $(\mathrm{OR}=1,67)$. $\mathrm{O}$ trabalho na limpeza urbana envolveu grande sobrecarga física, com pressão de tempo para sua execução. Conclusão: evidenciou-se a necessidade de medidas para redução do manuseio de cargas e de mudanças na organização do trabalho, incluindo pausas durante a jornada e adequação das tarefas ao tempo disponível, a fim de evitar sobrecargas física e psicológica dos trabalhadores.

Palavras-chave: dor; exposição ocupacional; extremidade inferior; limpeza urbana; saúde do trabalhador.

\begin{abstract}
Objective: to describe the characteristics of the urban cleaning workers' job and investigate the prevalence and factors associated with pain in their lower limbs. Methods: cross-sectional study carried out in Bahia, Brazil. Data collected between 2009 and 2010. Pain was assessed using the Nordic Musculoskeletal Questionnaire, while psychosocial demands at work were measured using the Job Content Questionnaire. Physical demands were also evaluated, including general postures and cargo handling. We used multiple logistic regression analysis to identify factors associated with lower limb pain. Results: 624 workers participated. There was a high prevalence of pain in the lower limbs in the last seven days $(23.7 \%)$ and in the last twelve months (42.1\%), being higher among cleaning workers and collectors. Lower limb pain was associated with the following: longer than three years working for the company $(O R=1.34)$; high exposure to cargo handling $(O R=1.35)$; psychological demand at work $(O R=1.87)$; and poor physical conditioning $(O R=1.67)$. Working at urban cleaning involved great physical overload, being produced under time pressure. Conclusion: The results evidenced the need for measures to reduce cargo handling and changes in work organization, including breaks during the day and adjusting tasks to the available time, in order to avoid workers' physical and psychological overload.
\end{abstract}

Keywords: pain; occupational exposure; lower extremity; urban cleaning; occupational health. 


\section{Introdução}

Os trabalhadores que manipulam lixo estão expostos a diversos riscos ocupacionais, tanto pelo contato direto com microrganismos quanto pelas condições físicas e psíquicas impostas ${ }^{1,2}$. Além disso, o manuseio do lixo pode gerar desgaste e desconforto em algumas regiões do corpo, decorrentes das altas demandas físicas dessa atividade ${ }^{3}$.

Na Bahia, o mecanismo de coleta do lixo é preponderantemente manual, exigindo força e dinamismo, pois requer que o trabalhador salte de diferentes níveis, corra e arremesse cargas, realizando movimentos repetitivos durante as horas trabalhadas ${ }^{3}$.

A associação entre o trabalho estático e cíclico e lesões em trabalhadores ${ }^{4}$ é mais relatada na literatura do que as consequências para a saúde relacionadas ao trabalho de alta exigência dinâmica que caracteriza algumas ocupações, como a dos trabalhadores da limpeza urbana (TLUs) ${ }^{5}$. Entretanto, esse tipo de trabalho pode ocasionar sobrecargas estruturais no corpo humano, desencadeando sintomas de dor que podem comprometer os membros inferiores (MMII), assim como outros segmentos corporais ${ }^{6,7}$. A dor nos membros inferiores pode ter origem, principalmente, no sistema musculoesquelético e/ou no sistema venoso ${ }^{8,9}$.

Os distúrbios musculoesqueléticos (DMEs) são de natureza multicausal - sendo o trabalho um dos possíveis determinantes para sua gênese e seu desfecho e figuram entre as principais causas de afastamento do trabalho, constituindo-se em relevante problema de saúde pública ${ }^{10}$. Diversos agravos incidem nos MMII e podem ocasionar sintomatologia dolorosa, como as degenerações articulares, tendíneas e musculares ${ }^{11}$. Outra etiologia da dor em MMII, além do comprometimento musculoesquelético, são os déficits no sistema vascular, caracterizados como insuficiência venosa nas pernas, especialmente referidos em mulheres, mas também verificados em trabalhadores ${ }^{9,11,12}$.

Evidências encontradas em estudos desenvolvidos em ambiente laboratorial, com população geral, sem queixas álgicas preexistentes, com o intuito de ressaltar sintomas de dor em MMII diante de atividades com manutenção da postura em pé, demonstraram que muitos sintomas em MMII se relacionam ao sistema vascular (aumento do fluxo sanguíneo, da temperatura da pele e circunferência da perna, além de fadiga muscular) ${ }^{12}$.

Dentre os fatores ocupacionais associados à dor em MMII estão: o manuseio de carga, especialmente o levantamento de peso; postura de trabalho em pé; deambulação constante; postura ajoelhada; agachamentos $^{12-14}$; e fatores psicossociais ${ }^{15}$.

Embora amplamente relatada no mundo do trabalho, referida como "dor nas pernas", entre homens e mulheres que trabalham predominantemente em pé $^{13}$, esta morbidade permanece pouco investigada. Messing, Tissot e Stock ${ }^{13}$, estudando "dor nas pernas” em uma população do Quebec, constataram uma associação entre a condição e as posturas de trabalho. Concordando com as autoras ${ }^{13}$, investigar a prevalência da dor em MMII, que causa sofrimento físico durante a jornada e limitações para o trabalho, impõe-se como agenda de pesquisa epidemiológica a fim de superar sua invisibilidade enquanto agravo relacionado ao trabalho. Esta perspectiva não é a da investigação clínica, que exige o arsenal propedêutico para diagnóstico etiológico da dor, mas sim da pesquisa epidemiológica do sintoma doloroso.

Como parte do projeto intitulado "Condições de Saúde e Trabalho em Trabalhadores de Limpeza Urbana”, este estudo buscou descrever as características do trabalho realizado na limpeza urbana e investigar a prevalência e os fatores que se associam à queixa de dor em MMII em TLUs.

\section{Métodos}

\section{Desenho e participantes}

Realizou-se estudo de corte transversal com todos os TLUs alocados em atividades de manutenção e de operação em empresa terceirizada responsável pelo recolhimento e transporte dos resíduos sólidos domiciliares na capital baiana, considerada a terceira mais populosa do Brasil em 2010. A carga horária de trabalho semanal dos pesquisados era de 44 horas e, dependendo da função, o trabalho era realizado no período diurno ou noturno. A população do estudo foi formada por 657 trabalhadores, todos do sexo masculino. Foram excluídos os trabalhadores de atividades administrativas e de gestão, a única mulher respondente, e os afastados.

A população de estudo incluiu trabalhadores dos setores de operação (coletor, motorista, agente de limpeza) e de manutenção (mecânico, auxiliar de controle e manutenção, auxiliar de serviços gerais, borracheiro, eletricista, lubrificador, pedreiro, pintor, soldador, vistoriador e lavador). O serviço de coleta domiciliar dos resíduos sólidos é feito por coletores e motoristas, que são responsáveis pelo trabalho diário de coleta e destinação final desses resíduos na estação de transbordo ou no aterro sanitário ${ }^{3}$. Os serviços complementares da limpeza urbana, como pintura de meios-fios, postes, viadutos e muros, limpeza de praias, capinagem e roçagem ${ }^{16}$, são realizados pelos agentes de limpeza. Os serviços de conservação da estrutura física da empresa, bem como dos caminhões compactadores utilizados na coleta dos resíduos ${ }^{16}$, são de incumbência dos trabalhadores da manutenção. 


\section{Coleta de dados}

A coleta de dados ocorreu entre dezembro de 2009 e abril de 2010. Anteriormente a essa etapa, realizou-se treinamento para aplicação do questionário e adequação das questões. Mestrandos e graduandos do curso de Fisioterapia de uma universidade pública realizaram as entrevistas com os trabalhadores, no início ou no fim da jornada, assegurando privacidade e sigilo das informações.

Para a realização da pesquisa foi utilizado questionário ${ }^{17}$ que continha questões acerca de: informações sociodemográficas (idade, escolaridade, raça/ cor da pele); tempo de vida laboral total, de trabalho na empresa, jornada de trabalho; realização de horas extras; demandas físicas (posturas gerais de trabalho e manuseio de cargas) e psicossociais no trabalho; condicionamento físico, excesso de peso (obtido por meio de medida direta de peso e altura para cálculo do Índice de Massa Corporal - IMC), tabagismo, uso de bebidas alcoólicas, trabalho doméstico; e sintomas dolorosos nos segmentos dos MMII.

\section{Variáveis do estudo}

Utilizou-se o Nordic Musculoskeletal Questionnaire $(\mathrm{NMQ})^{18}$, instrumento validado no Brasil e amplamente utilizado para mensurar sintomas dolorosos em diversos segmentos corporais. Esse instrumento visa a mensurar o sintoma da dor e não sua etiologia. Descreveu-se a dor em MMII nos segmentos coxa, joelho, perna e tornozelo/pé, nos doze meses e nos sete dias prévios à aplicação do questionário. Na etapa descritiva do estudo foram apresentadas as prevalências de dor em cada um dos diferentes segmentos dos MMII e na região corporal como um todo. Na etapa analítica, adotou-se como variável dependente o relato de dor em MMII nos últimos doze meses. Considerou-se como variáveis independentes o tempo de trabalho na empresa, a extensão da jornada semanal de trabalho, as horas extras, as demandas física e psicossocial no trabalho, assim como as variáveis sociodemográficas e extraocupacionais.

A variável tempo de trabalho na empresa, que corresponde ao período na função, foi descrita em meses e estratificada na mediana. A jornada de trabalho teve como estrato de risco os indivíduos com jornada semanal maior que 44 horas. A variável hora extra foi dicotomizada (presente ou não).

A demanda física foi avaliada por meio de questões que mensuraram as posturas assumidas durante o trabalho (andando, correndo, saltando do caminhão) e o manuseio de carga (levantar, empurrar e puxar). Os itens foram medidos em escalas de duração de seis pontos, com âncoras nas extremidades (sendo $0=$ nunca, e $5=$ o tempo todo). Para fins de análise, os dados foram dicotomizados com corte na mediana. As questões de demanda física utilizadas tiveram sua dimensionalidade, bem como sua confiabilidade, avaliada (validade de construto) em estudo que incluiu esta população de TLU. Tanto a análise da validade estrutural quanto o teste-reteste mostraram resultados que indicam o uso das questões ${ }^{19}$.

As demandas psicossociais (demanda, controle e apoio social) no trabalho foram mensuradas por meio do Job Content Questionnaire (JCQ), versão validada e traduzida para o português ${ }^{20}$. Para averiguar os itens de demanda psicológica e controle no trabalho, os TLUs responderam a nove perguntas para cada item, sendo a dimensão controle no trabalho relacionada às habilidades e autoridade na tomada de decisão. A dimensão demanda psicológica está associada às exigências requeridas na realização das tarefas, envolvendo pressão de tempo, nível de concentração, interrupção das tarefas e necessidade de esperar o trabalho de outros na equipe. A dimensão apoio social no trabalho incorpora o apoio do supervisor e de colegas durante a realização do trabalho. No estudo, essas variáveis foram estratificadas na mediana e, na fase descritiva do estudo, foram apresentadas tanto separadamente quanto combinadas em uma variável que classificou como alta exposição psicossocial quando se satisfaziam pelo menos duas das seguintes condições: alta demanda, baixo controle e baixo suporte social. Na fase analítica, controle, demanda e apoio social foram incluídos separadamente na modelagem.

As características sociodemográficas analisadas foram idade (corte na mediana, sendo o estrato de exposição aqueles com idade $\geq 33$ anos) e escolaridade, divididos em dois estratos (o estrato de risco foi composto por aqueles com pelo menos o ensino médio completo), e as variáveis extraocupacionais foram condicionamento físico (escala de 0 a 5, estratificada em: 0-3 = condicionamento insuficiente e 4-5 = condicionamento suficiente), IMC (entre $\geq 25$ $\mathrm{e} \geq 30=$ sobrepeso ou obesidade e entre $<18,5 \mathrm{e}$ $<25=$ peso normal ou baixo), tabagismo (uso de tabaco ou não), etilismo (risco: uso de bebida uma a três vezes por semana; referência: nunca bebeu, parou de beber há mais de um ano ou bebe de uma a três vezes/mês) e trabalho doméstico, corte na mediana, expostos ( $\geq 2$ horas na última semana).

\section{Análises estatísticas}

Os resultados descritivos são apresentados de acordo com a ocupação (coletores, agentes de limpeza, motoristas e trabalhadores da manutenção), caracterizando a população, as variáveis de exposição ocupacional e a prevalência de dor em MMII e seus segmentos. 
Para apresentar as demandas físicas no trabalho, foram confeccionados boxplots (diagramas de caixa) ${ }^{21}$ para os quatro grupos ocupacionais. Os diagramas apresentam a distribuição da exposição às demandas físicas por grupo: a) coletores, b) motoristas, c) agentes de limpeza e d) pessoal da manutenção. As demandas físicas apresentadas se referem à postura geral de trabalho: 1) trabalho em pé, 2) sentado, 3) correndo, 4) andando, 5) saltando, 6) agachado; ou manuseio de carga: 7) levantar, 8) empurrar e 9) puxar. Todas as demandas foram mensuradas em escala numérica de seis pontos (0-5), com âncoras nas extremidades. A distribuição da exposição para cada demanda física é mostrada em gráficos, que apresentam, no eixo x, os quatro grupos de exposição (a-d) e, no eixo y, a escala de resposta para cada demanda mensurada (0-5 pontos).

A análise multivariável para identificação dos fatores associados à dor em MMII foi conduzida por meio da regressão logística. A seleção das variáveis independentes teve como critério a plausibilidade fisiopatológica, teórica e epidemiológica das associações, a partir das evidências mais consistentes encontradas na literatura. Com base nesse critério, inicialmente entraram no modelo múltiplo: idade, escolaridade, raça/cor da pele, jornada de trabalho, hora extra, tempo de trabalho na empresa, manuseio de carga (levantar, puxar, empurrar), trabalho dinâmico (correndo, saltando, andando), demanda psicológica, controle no trabalho, apoio social, trabalho doméstico, excesso de peso, condicionamento físico, uso de álcool e tabagismo. As variáveis com magnitude de associação (odds ratio - OR) igual ou acima de $30 \%$ (OR $\geq 1,30$ ou $\mathrm{OR} \leq 0,70$ ) foram mantidas no modelo final ${ }^{22}$. Desta forma, a OR, medida de associação fornecida na regressão logística, é apresentada para cada variável do modelo final.

No estudo, foram adotados procedimentos compatíveis com a natureza não aleatória da população investigada, estatística analítica (OR) não seguida de estatística inferencial (intervalos de confiança ou valor de p), conforme Silvany Neto $^{21}$ e outras publicações sobre o tema ${ }^{23-26}$.

Os dados foram analisados utilizando o programa estatístico software R versão 3.0.3 (2014-03-06).

\section{Considerações éticas}

O estudo foi aprovado pelo Comitê de Ética em Pesquisa do Hospital São Rafael, protocolo n ${ }^{\circ} 48 / 09$, em 28 de outubro de 2009. Todos os participantes assinaram o termo de consentimento livre e esclarecido.

\section{Resultados}

Dos 657 trabalhadores, participaram 624 (taxa de resposta de 95\%), todos do sexo masculino, com média de 33,9 anos de idade. Entre os grupos, 50,9\% dos motoristas tinham idade acima de 39 anos, enquanto $60,2 \%$ dos coletores estavam abaixo de 32 anos (com mediana em 30 anos), e apenas 18\% tinham 39 anos de idade ou mais.

A população dos TLUs apresentou escolaridade inferior ao ensino médio completo, principalmente entre coletores e agentes de limpeza, destacando-se os motoristas com maior nível de escolaridade. Mais de $60 \%$ dos TLUs relataram condicionamento físico suficiente, mas apenas 35,6\% dos motoristas tiveram essa percepção. O tempo de trabalho na empresa e o de vida laboral total tiveram mediana de 36 meses e 19 anos, respectivamente. Coletores e motoristas apresentaram tempo na empresa (função) menor que 3 anos. A jornada semanal dos TLUs foi superior a 44 horas, prevalecendo a realização de horas extras extras, principalmente entre motoristas e coletores, constatada tanto pela alta frequência de horas extras quanto pela média de horas semanais trabalhadas (Tabela 1).

Na Tabela 2, descrevem-se as frequências absolutas e relativas dos aspectos psicossociais do trabalho para cada grupo ocupacional, em que são vistas as frequências de baixo controle, alta demanda, baixo apoio social e alta exposição psicossocial, combinando as três dimensões. A alta demanda psicológica e o baixo controle prevaleceram, principalmente entre os coletores. Os motoristas tiveram mais controle no trabalho quando comparados aos coletores. O baixo apoio social entre os grupos teve prevalência semelhante.

Na Figura 1, os diagramas de caixa (boxplots) mostram a distribuição das respostas de 0 a 5 para cada demanda física (eixo y), segundo o grupo ocupacional (eixo x). Observa-se que os coletores apresentaram maiores valores típicos para quase todas as demandas físicas, com concentração de respostas nos extremos da escala, marcando 5 para trabalho em pé e correndo, representando a adoção dessas posturas durante todo o tempo da jornada, e 0 para trabalho sentado, significando a não adoção dessa postura durante a jornada. Além disso, é possível observar que o manuseio de carga ocorre durante quase todo o tempo da jornada de trabalho dos coletores, como se vê para as variáveis levantar (primeiro quartil já no ponto 4 da escala e mediana no 5), empurrar e puxar cargas (ambas com mediana no ponto 4 da escala de resposta). As atividades saltando e correndo foram mais predominantes entre os coletores. Ressalta-se que o trabalho dos coletores e agentes de limpeza exige estar em pé durante quase toda a jornada, enquanto o gráfico de trabalho sentado mostra, de forma consistente, que esses trabalhadores nunca ou quase nunca se sentam durante a jornada, com resultados concentrados na extremidade 0 da escala de respostas. Os agentes de limpeza e pessoal de 
manutenção apresentam maior exposição ao trabalho andando. Os motoristas alcançaram os maiores valores na posição sentada, conforme esperado para essa ocupação. Acrescenta-se que, para quase todas as demandas físicas, as assimetrias prevaleceram, caracterizando os extremos da exposição física dos TLUs. Observa-se alguma variabilidade da exposição entre os agentes de limpeza.

Tabela 1 Dados sociodemográficos, características ocupacionais e extraocupacionais, segundo grupo ocupacional, em trabalhadores da limpeza urbana, Salvador, Bahia, 2010

\begin{tabular}{|c|c|c|c|c|c|}
\hline & \multicolumn{5}{|c|}{ Grupos de ocupação } \\
\hline & $\begin{array}{c}\text { Coletores } \\
n=367 \\
(58,8 \%)\end{array}$ & $\begin{array}{c}\text { Motoristas } \\
n=118 \\
(18,9 \%)\end{array}$ & $\begin{array}{c}\text { Agentes de } \\
\text { limpeza } \\
n=87 \\
(13,9 \%)\end{array}$ & $\begin{array}{c}\text { Pessoal da } \\
\text { manutenção } \\
n=52 \\
(8,3 \%)\end{array}$ & $\begin{array}{c}\text { Total } \\
n=624 \\
(100,0 \%)\end{array}$ \\
\hline \multirow{2}{*}{ Variáveis } & \multicolumn{5}{|c|}{ Frequências } \\
\hline & $n(\%)$ & $n(\%)$ & $n(\%)$ & $n(\%)$ & $n(\%)$ \\
\hline \multicolumn{6}{|l|}{ Idade* } \\
\hline$<28$ & $123(33,5)$ & $7(6,0)$ & $9(10,3)$ & $9(17,3)$ & $148(23,8)$ \\
\hline $28-32$ & $98(26,7)$ & $17(14,7)$ & $25(28,7)$ & $12(23,1)$ & $152(24,5)$ \\
\hline $33-38$ & $79(21,5)$ & $33(28,4)$ & $31(35,6)$ & $12(23,1)$ & $155(24,9)$ \\
\hline$\geq 39$ & $67(18,3)$ & $59(50,9)$ & $22(25,4)$ & $19(36,5)$ & $167(26,8)$ \\
\hline \multicolumn{6}{|l|}{ Raça/Cor da pele* } \\
\hline Preta & $236(64,5)$ & $40(33,9)$ & $48(55,2)$ & $20(39,2)$ & $344(55,3)$ \\
\hline Parda & $110(30,1)$ & $60(50,8)$ & $35(40,2)$ & $27(52,9)$ & $232(37,3)$ \\
\hline Branca & $20(5,4)$ & $18(15,3)$ & $4(4,6)$ & $4(7,9)$ & $46(7,4)$ \\
\hline \multicolumn{6}{|l|}{ Escolaridade* } \\
\hline$<$ ensino médio completo & $273(74,6)$ & $31(26,3)$ & $60(69,8)$ & $28(54,9)$ & $392(63,1)$ \\
\hline$\geq$ ensino médio completo & $93(25,4)$ & $87(73,7)$ & $26(30,2)$ & $23(45,1)$ & $229(36,9)$ \\
\hline \multicolumn{6}{|l|}{ Hora extra } \\
\hline Sim & $343(93,5)$ & $117(99,2)$ & $37(42,5)$ & $34(65,4)$ & $531(85,1)$ \\
\hline Não & $24(6,5)$ & $1(0,8)$ & $50(57,5)$ & $18(34,6)$ & $93(14,9)$ \\
\hline \multicolumn{6}{|l|}{ Jornada semanal* } \\
\hline$>44$ horas & $311(84,7)$ & $106(89,8)$ & $59(70,2)$ & $39(72,2)$ & $517(82,9)$ \\
\hline$\leq 44$ horas & $56(15,3)$ & $12(10,2)$ & $25(29,8)$ & $15(27,8)$ & $107(17,1)$ \\
\hline \multicolumn{6}{|l|}{ Vida laborativa*" } \\
\hline$\geq 19$ anos & $147(40,4)$ & $84(71,8)$ & $50(57,5)$ & $31(59,6)$ & $312(50,3)$ \\
\hline$<19$ anos & $217(59,6)$ & $33(28,2)$ & $37(42,5)$ & $21(40,4)$ & $308(49,7)$ \\
\hline \multicolumn{6}{|l|}{ Condicionamento físico } \\
\hline Insuficiente & $132(36,0)$ & $76(64,4)$ & $43(49,4)$ & $26(50,0)$ & $277(44,4)$ \\
\hline \multirow[t]{2}{*}{ Suficiente } & $235(64,0)$ & $42(35,6)$ & $44(50,6)$ & $26(50,0)$ & $347(55,6)$ \\
\hline & Média (DP**) & Média (DP***) & Média $\left(D P^{* *}\right)$ & Média (DP***) & Média $\left(D P^{* * *}\right)$ \\
\hline Idade $^{*}$ & $31,8(7,97)$ & $38,9(7,64)$ & $34,9(6,69)$ & $36,0(8,94)$ & $33,9(8,33)$ \\
\hline \multirow[t]{2}{*}{ Jornada semanal* } & $55,7(14,3)$ & $59,6(13,1)$ & $47,1(9,0)$ & $49,6(11,0)$ & $54,8(13,8)$ \\
\hline & Mediana & Mediana & Mediana & Mediana & Mediana \\
\hline Vida laborativa* (em anos) & 16,0 & 23,0 & 19,0 & 20,5 & 19,0 \\
\hline Tempo na empresa (em meses) & 35,0 & 26,5 & 59 & 38 & 36,0 \\
\hline
\end{tabular}

* Os valores registrados são dados válidos para cada variável. Dados ignorados foram evidenciados por meio do registro de números absolutos.

*** DP: desvio padrão. 
Tabela 2 Frequência de exposição psicossocial no trabalho, segundo grupo ocupacional, em trabalhadores da limpeza urbana, Salvador, Bahia, 2010

\begin{tabular}{lccccc}
\hline & \multicolumn{5}{c}{ Grupos de ocupação } \\
\cline { 2 - 6 } & $\begin{array}{c}\text { Coletores } \\
n=367\end{array}$ & $\begin{array}{c}\text { Motoristas } \\
n=118\end{array}$ & $\begin{array}{c}\text { Agentes de } \\
\text { limpeza } \\
n=87\end{array}$ & $\begin{array}{c}\text { Pessoalda } \\
\text { manutenção } \\
n=52\end{array}$ & $\begin{array}{c}\text { Total } \\
n=624\end{array}$ \\
\hline Variáveis & $n(\%)$ & $n(\%)$ & $n(\%)$ & $n(\%)$ & $n(\%)$ \\
\hline Baixo controle & $198(54,5)$ & $31(26,7)$ & $40(46,5)$ & $8(15,4)$ & $277(44,9)$ \\
Alta demanda psicológica & $215(60,7)$ & $59(50,0)$ & $32(38,6)$ & $12(23,5)$ & $318(52,5)$ \\
$\begin{array}{l}\text { Baixo apoio social } \\
\text { Alta exposição psicossocial } \\
\text { no trabalho }\end{array}$ & $163(44,8)$ & $55(46,6)$ & $29(33,7)$ & $22(43,1)$ & $269(43,5)$ \\
\hline
\end{tabular}
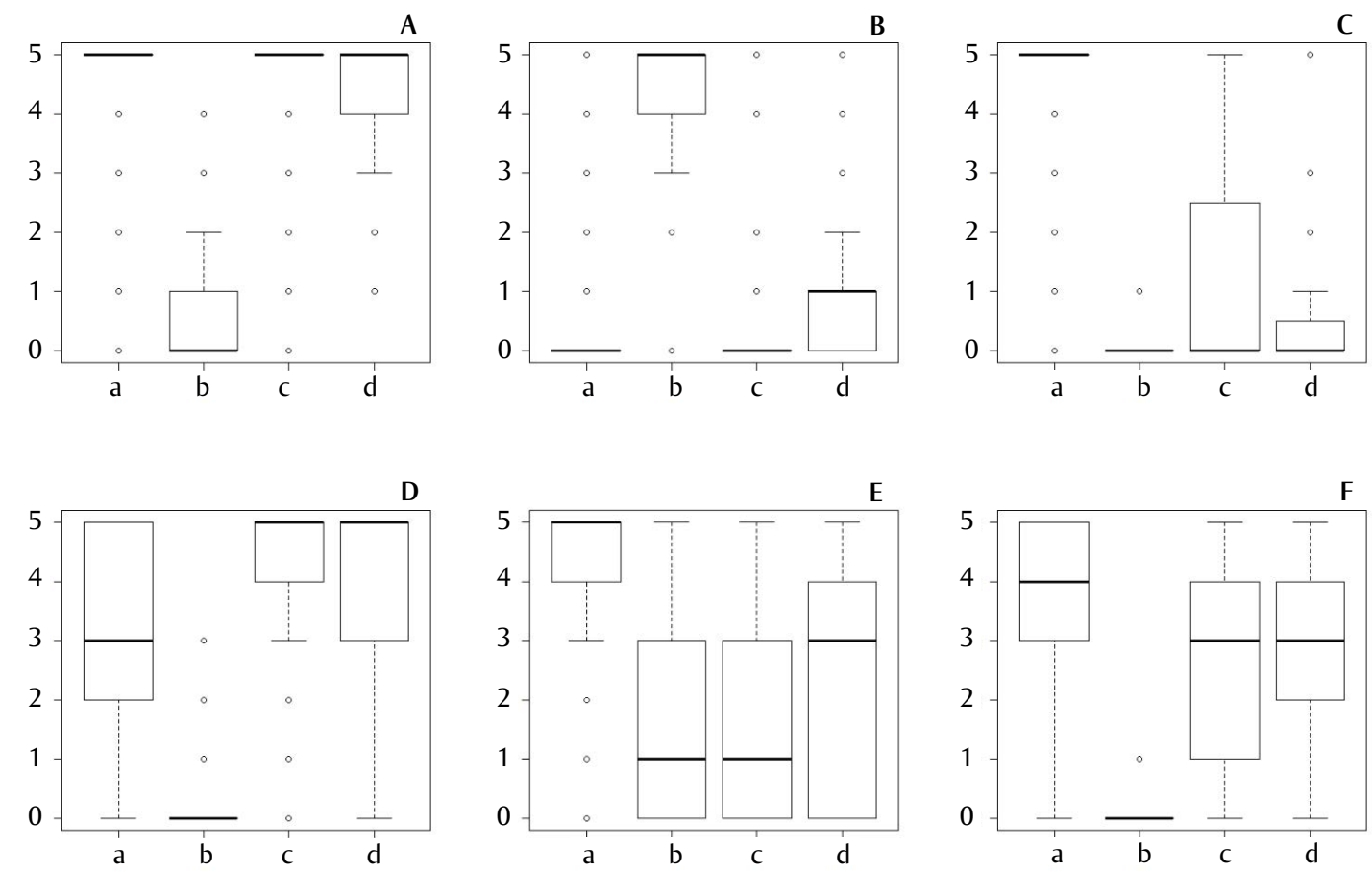

G
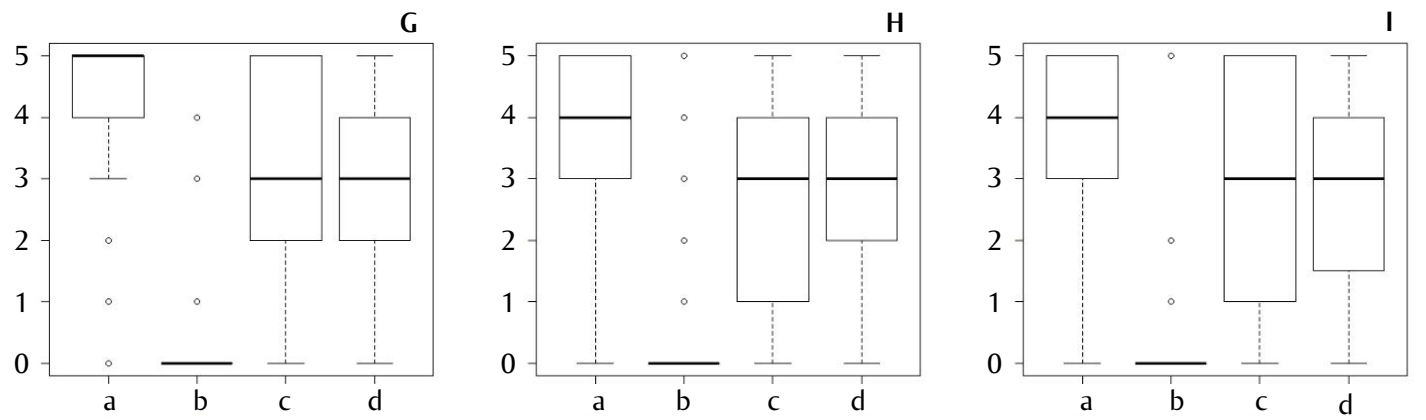

Eixo y: escala de resposta das variáveis de demanda física (0-5): (0) nunca a (5) o tempo todo.

Eixo x: ocupação: a) coletores; b) motoristas; c) agentes de limpeza; e d) pessoal da manutenção.

A: trabalho em pé; B: sentado; C: correndo; D: andando; E: saltando; F: agachado, G: levantando carga; H: empurrando carga; I: puxando carga.

Figura 1 Distribuição das demandas físicas por grupo ocupacional dos trabalhadores da limpeza urbana, Salvador, Bahia, 2010 
Na Tabela 3, descrevem-se as prevalências de dor em MMII e seus segmentos, na qual se observa que a dor em MMII nos últimos sete dias foi de $23,7 \%(\mathrm{n}=148)$ e em doze meses foi de $42,1 \%$ $(\mathrm{n}=263)$. Os agentes de limpeza e os coletores apresentaram maiores prevalências de dor em MMII, nos últimos doze meses, quando comparados aos motoristas e trabalhadores da manutenção. Em relação à dor referida nos segmentos coxa/joelho e perna, observou-se maior prevalência entre agentes de limpeza e coletores, tanto nos sete dias quanto nos doze meses anteriores à aplicação do questionário. Para o segmento tornozelo/pé, os coletores relataram mais dor nos doze meses anteriores, quando comparados aos demais grupos.
A Tabela 4 apresenta as associações brutas e ajustadas entre as variáveis independentes e dor em MMII, nos últimos doze meses. A partir dos dados ajustados, verificou-se que trabalhadores expostos à alta demanda psicológica no trabalho apresentaram mais dor em MMII $(\mathrm{OR}=1,87)$ quando comparados aos menos expostos a essa demanda. Ademais, apresentaram sintoma de dor aqueles que tinham um tempo de trabalho maior na empresa (OR $=1,34)$ e os trabalhadores submetidos ao elevado manuseio de carga, com 35\% mais chance de dor que aqueles expostos a um trabalho com baixo manuseio de carga. Os TLUs com condicionamento físico insuficiente apresentaram maiores chances de relatar dor em MMII, quando comparados àqueles com condicionamento suficiente $(\mathrm{OR}=1,67)$.

Tabela 3 Prevalência (\%) de dor em membros inferiores e seus segmentos, segundo grupo ocupacional e população total, em trabalhadores da limpeza urbana, Salvador, Bahia, 2010

\begin{tabular}{|c|c|c|c|c|c|}
\hline \multirow[b]{2}{*}{ Período/Segmento } & \multicolumn{5}{|c|}{ Grupos de ocupação } \\
\hline & $\begin{array}{c}\text { Coletores } \\
n=367\end{array}$ & $\begin{array}{c}\text { Motoristas } \\
n=118\end{array}$ & $\begin{array}{l}\text { Agentes de Limpeza } \\
\qquad n=87\end{array}$ & $\begin{array}{c}\text { Pessoal da } \\
\text { manutenção } \\
n=52\end{array}$ & $\begin{array}{c}\text { Total } \\
n=624\end{array}$ \\
\hline Últimos sete dias & $n(\%)$ & $n(\%)$ & $n(\%)$ & $n(\%)$ & $n(\%)$ \\
\hline Membros inferiores & $82(22,3)$ & $27(22,9)$ & $27(31,0)$ & $12(23,1)$ & $148(23,7)$ \\
\hline Coxa/Joelho & $49(13,4)$ & $13(11,0)$ & $20(23,0)$ & $7(13,5)$ & $89(14,3)$ \\
\hline Perna & $37(10,1)$ & $10(8,5)$ & $10(11,5)$ & $5(9,6)$ & $62(9,9)$ \\
\hline Tornozelo/Pé & $24(6,5)$ & $8(6,8)$ & $5(5,7)$ & $3(5,8)$ & $40(6,4)$ \\
\hline Últimos doze meses & $n(\%)$ & $n(\%)$ & $n(\%)$ & $n(\%)$ & $n(\%)$ \\
\hline Membros inferiores & $158(43,1)$ & $42(35,6)$ & $43(49,4)$ & $20(38,5)$ & $263(42,1)$ \\
\hline Coxa/Joelho & $95(25,9)$ & $28(23,7)$ & $32(36,8)$ & $10(19,2)$ & $165(26,4)$ \\
\hline Perna & $78(21,3)$ & $18(15,3)$ & $21(24,1)$ & $10(19,2)$ & $127(20,4)$ \\
\hline Tornozelo/Pé & $43(11,7)$ & $11(9,3)$ & $8(9,2)$ & $5(9,6)$ & $67(10,7)$ \\
\hline
\end{tabular}

Tabela 4 Análise bruta e ajustada dos fatores associados à dor em membros inferiores em trabalhadores da limpeza urbana, Salvador, Bahia, $2010(\mathrm{n}=583)$

\begin{tabular}{lcc}
\hline Variáveis independentes & $\begin{array}{c}\text { OR } \\
\text { bruta }\end{array}$ & $\begin{array}{c}\text { OR } \\
\text { ajustada }\end{array}$ \\
\hline Demanda psicológica no trabalho & & \\
$\quad$ Alta & 1,86 & 1,87 \\
$\quad$ Baixa & 1,00 & 1,00 \\
Manuseio de carga & & \\
$\quad$ Alta exposição & 1,35 & 1,35 \\
$\quad$ Baixa exposição & 1,00 & 1,00 \\
Tempo de trabalho na empresa & & \\
$\quad \geq 36$ meses & 1,28 & 1,34 \\
$\quad<36$ meses & 1,00 & 1,00 \\
Condicionamento físico & & \\
$\quad$ Insuficiente & 1,54 & 1,67 \\
$\quad$ Suficiente & 1,00 & 1,00 \\
\hline
\end{tabular}

OR: odds ratio. 


\section{Discussão}

\section{Características da população trabalhadora e do trabalho na limpeza urbana}

Os TLUs participantes do estudo são, na maioria, indivíduos de raça/cor da pele preta, todos do sexo masculino, com baixa escolaridade e elevada jornada semanal de trabalho. Outros estudos realizados com trabalhadores desta categoria profissional corroboram os resultados encontrados aqui referentes à idade, sexo e escolaridade ${ }^{2,5,27}$. As características evidenciadas podem revelar um trabalho pouco reconhecido socialmente e de grande exigência física, o que pode explicar a maior presença de trabalhadores menos escolarizados, de raça/cor preta, que têm maior dificuldade de inserção em profissões prestigiadas, além de trabalhadores jovens, supostamente tolerantes ao trabalho físico extenuante, a exemplo dos coletores. A própria Classificação Brasileira de Ocupações (CBO) ${ }^{28}$ aponta como requisitos, para as profissões de gari e coletor, ensino fundamental incompleto, preparo físico e agilidade ${ }^{28}$, implicando na seleção de jovens saudáveis fisicamente para execução do trabalho pesado. A descrição da CBO contradiz o que a Ergonomia preconiza como exigência para compatibilizar trabalho e saúde: condições de trabalho adequadas, menos onerosas e não adaptação do trabalhador ao trabalho adverso ${ }^{29,30}$.

Um estudo conduzido com trabalhadores da mesma população revela estratégias de proteção adotadas pelos coletores ao longo da jornada de trabalho, a fim de evitar agravos, revelando um saber adquirido na prática ${ }^{3}$. Esses métodos de antecipação incluem, entre outros, a "estratégia de redução do lixo”, na qual a prática de balançar os sacos de lixo permite detectar material perfurocortante e evitar acidentes ${ }^{31}$.

Boa parte dos TLUs tinha menos de três anos de trabalho na empresa, no entanto mais de 50\% deles apresentavam vida laboral total de 19 anos ou mais, fato que, além de indicar tempo curto de permanência na limpeza urbana, pode evidenciar também a inserção precoce desses trabalhadores no mercado de trabalho, considerando que metade dos coletores tem idade inferior a 30 anos. A jornada excessiva e as constantes extrapolações das horas trabalhadas são possíveis fatores de dano à saúde ${ }^{2}$. Para esses trabalhadores, a quantidade de lixo nas vias públicas determina o ritmo e o tempo do trabalho, comprometendo as pausas reais para descanso corporal e redução da fadiga psíquica ${ }^{3}$.

Os TLUs estão submetidos a alta demanda psicológica, principalmente coletores e motoristas, responsáveis pela coleta de lixo nas ruas ${ }^{3}$, atividade que requer estado de alerta, haja vista o grande fluxo de veículos, exigindo atenção e agilidade ${ }^{28}$ a fim de evitar acidentes ${ }^{1}$. Além disso, existe pressão para não ultrapassar a jornada de trabalho, apesar da necessidade de maior tempo para coleta, especialmente em dias após final de semana e festas ${ }^{3}$. Contraditoriamente, são cobrados pelo zelo dos equipamentos de trabalho, a exemplo do caminhão, que não deve ser sobrecarregado com lixo, tornando necessário realizar maior número de viagens ao aterro para reduzir a carga do veículo e, consequentemente, resultando em uma jornada mais longa. Contradições como esta podem implicar alta demanda psicológica para os trabalhadores ${ }^{3}$.

Um achado relevante deste estudo foi o baixo controle sobre o trabalho entre os coletores e os agentes de limpeza em relação, especialmente, aos motoristas, com os quais os coletores compartilham o trabalho nas ruas. Constatou-se uma diferença considerável entre essas ocupações no que se refere ao grau de autonomia e de liderança, aspectos avaliados pela dimensão de controle do modelo demanda-controle $^{20}$. Os motoristas são os líderes da equipe, usufruindo de maior poder de decisão, inclusive estando no comando dos coletores, aos quais impõem sua autoridade conferida pela empresa ${ }^{3}$. Portanto, a exposição às demandas psicossociais dos TLUs não é homogênea, considerando os resultados descritos de acordo com os quatro grupos ocupacionais.

Um estudo realizado com coletores de lixo no Irã evidenciou prevalências similares relacionadas à alta exposição às demandas psicológicas no trabalho, destacando que 56\% dos coletores apresentaram baixo controle no trabalho. Verificou-se também que o controle no trabalho era inversamente proporcional à escolaridade dos TLUs ${ }^{2}$, dado que diverge do encontrado neste estudo, no qual os motoristas tinham maior escolaridade e controle no trabalho, enquanto os coletores tinham menor escolaridade e controle.

Além dos achados sobre a exposição psicossocial, é importante discutir as características do trabalho na limpeza urbana, de marcante natureza física ${ }^{2}$. Para o exercício das tarefas, o corpo do trabalhador, muitas vezes, funciona como ferramenta para o trabalho braçal predominante. Essa demanda física foi evidenciada, conforme os quatro grupos de ocupação.

A alta exigência física entre os coletores foi verificada durante quase todo o tempo de jornada, fato explicado pela permanência desses trabalhadores em atividades de manuseio de cargas, adotando posturas anômalas nesse período. Ademais, as longas jornadas implicam tempo insuficiente para recuperação do desgaste físico, favorecendo quadros de dor.

Outro estudo realizado com trabalhadores de resíduos sólidos no Irã ${ }^{5}$ também encontrou dados semelhantes de exposição às demandas físicas e psíquicas 
no trabalho dos coletores, entre elas a pressão temporal para a realização da coleta e as repetições nas atividades de levantamento de carga.

Neste estudo, os coletores experimentam tensão excessiva constante para manutenção postural dos desalinhamentos no tornozelo/pé, perna e coxa/joelho, diante dos diversos movimentos requeridos em um dia de trabalho. Os princípios da biomecânica elucidam que a ausência do alinhamento neutro das articulações gera desequilíbrio em tendões, ligamentos e músculos que, além de comprometer sua função, pode ocasionar dor ${ }^{32}$.

A presença de movimentos corporais rápidos condiciona os músculos a altos picos de tensão, provocando fadiga muscular, quando deveria haver períodos de baixa intensidade, intercalado com pausas frequentes, já que é um trabalho extenuante ${ }^{32}$. A partir dessa situação de trabalho, em que os TLUs realizam o deslocamento rápido do corpo ao mesmo tempo em que carregam sacolas, baldes e caixas de lixo pesados e os arremessam no caminhão, além de saltos na subida e descida do estribo, pode-se explicar a dor referida em MMII e, particularmente, em tornozelos e pé.

Outro fator de destaque é a estabilização dinâmica contínua de grupos musculares ${ }^{32}$ para empurrar contêineres carregados de lixo e fixá-los no caminhão, ocasionando gasto energético, implicando fadiga muscular e déficit no suprimento sanguíneo para repor nutrientes aos músculos, acumulando metabólitos e culminando em sobrecarga muscular $\mathrm{e}$, consequentemente, dor.

\section{Dor em MMII e as demandas do trabalho}

Encontrou-se alta morbidade em membros inferiores entre os TLUs, sendo mais evidente entre agentes de limpeza e coletores para os últimos doze meses. Os achados de alta prevalência de sintomas dolorosos em MMII estão em consonância com estudos realizados com TLUs no Brasil e em outros países ${ }^{5,7,8}$.

Trata-se de população jovem que relata presença frequente de dor na semana anterior de trabalho e nos últimos doze meses e, a despeito da queixa, permanece nos postos de trabalho, exposta aos fatores ocupacionais descritos. A dor que os trabalhadores são instados a suportar cotidianamente favorece distúrbios em MMII, agravados no decorrer do tempo, levando à incapacidade para as atividades ocupacionais ${ }^{5,33}$.

Além do absenteísmo no trabalho, a busca por serviços de saúde é uma das consequências dos $\mathrm{DMEs}^{33}$. No Irã, $35 \%$ dos coletores pesquisados procuraram tratamento da dor musculoesquelética,
$20 \%$ faltaram o trabalho e $53 \%$ relataram que podem deixar o trabalho futuramente por conta dos distúrbios musculoesqueléticos ${ }^{5}$.

A presença de movimentos articulares frequentes em joelhos são características físicas da ocupação dos TLUs, principalmente de agentes de limpeza e coletores, que tiveram, respectivamente, mais registros de dor nesse segmento. Enquanto estes realizam saltos e agachamentos, aqueles permanecem muito tempo agachado. A literatura aponta que tarefas que associam levantamento de peso com agachamentos e ocupações com alta exigência física estão associadas a desordens no joelho ${ }^{14}$.

Na população geral, a queixa de dor nas pernas entre os homens apresenta menor magnitude do que entre as mulheres, sendo resultado da presença de fatores de risco para doença venosa intrínseca ao sexo feminino ${ }^{34}$. Contudo, um estudo em laboratório verificou maior aumento do volume da perna em homens, quando comparados às mulheres, devido ao edema gerado durante a realização de atividade em pé, sentada e com alternância dessas posturas ${ }^{35}$.

No segmento dos tornozelos e pés, a literatura ressalta que as posturas assumidas no trabalho são desencadeadoras de dor. Um estudo realizado com participantes em laboratório por Lin et al..$^{36}$ detectou que a permanência da postura em pé, sem pausas, o tipo de calçado e as superfícies rígidas geram dor no pé. Ainda, a literatura aponta haver uma relação entre o trabalho andando - como o realizado pelos coletores participantes deste estudo, com constantes deslocamento e deambulação - com o aumento da pressão dos músculos plantares e baixo limiar para os sintomas vasculares ${ }^{37}$. Entretanto, embora a prevalência seja mais alta entre coletores, é importante ressaltar que a morbidade por segmentos das extremidades distais está presente em todos os grupos ocupacionais dos TLUs.

As análises mostraram associação entre alta demanda psicológica no trabalho dos TLUs com dor em MMII, e outras investigações realizadas com essa população apontam uma relação do trabalho fisicamente exigente com dor em $\mathrm{MMII}^{5,8}$.

Os resultados de pesquisas em ambientes de trabalho, visando a identificar fatores que se associam à dor nos MMII, vão ao encontro dos achados deste estudo $^{7,8}$. Entre TLUs no Egito, o alto controle no trabalho e o curto período de atividade como coletor foram fatores de proteção contra sintomas musculoesqueléticos. No entanto, o manuseio de cargas acima de $20 \mathrm{~kg}$ e a deambulação frequente foram fortemente associados com sintomas de dor ${ }^{8}$.

Em estudo realizado no Irã, a demanda física (elevar, puxar, empurrar, andar, subir e descer do estribo), a demanda psicológica e o controle no trabalho foram 
associados à dor musculoesquelética ${ }^{5}$. Neste estudo, o tempo de trabalho na empresa maior que 36 meses, ou seja, três anos de atividade laborativa como TLU, e a alta exposição ao manuseio de carga aumentaram em 35\% a chance de dor em MMII entre os trabalhadores. Por outro lado, entre os fatores psicossociais, não se evidenciou o papel protetor do alto grau de controle, mas a alta demanda psicológica aumentou em $87 \%$ a prevalência de dor em MMII.

É conhecido que o trabalho com alta exigência psicológica pode ocasionar o adoecimento psíquico dos indivíduos ${ }^{15}$, mas ainda se discute o seu papel na causalidade dos sintomas físicos entre os trabalhadores.

Diversas teorias buscam explicar os mecanismos psicossociais que levam à dor musculoesquelética ${ }^{38}$ em trabalhadores. Trabalho com alta demanda psicológica e baixo controle contribui para ocorrência dos distúrbios musculoesqueléticos em membros superiores. É possível que tal exposição possa explicar a dor em membros inferiores dos TLUs, cujo labor é marcado fortemente por baixo controle e alta demanda.

Os fatores psicossociais podem agir direta ou indiretamente no sistema musculoesquelético e, consequentemente, podem exercer um papel na dor em MMII, de natureza musculoesquelética. Também podem elevar a atividade muscular do trabalhador, que, para responder às exigências das tarefas, exacerba sua capacidade interna individual, adota posturas inadequadas ou se submete a mais tempo de exposição para concluir as tarefas, gerando cargas mecânicas anormais no corpo. Os mecanismos de defesa do corpo também podem interpretar as demandas requeridas como ameaça e desencadear uma cascata fisiológica de resposta ao estresse, tais como incremento do tônus muscular e redução da circulação local ${ }^{39}$. Assim, marcadores fisiológicos específicos são ativados e explicam o papel das demandas psicossociais na dor musculoesquelética.

Neste estudo, os TLUs que relataram condicionamento físico insuficiente tiveram $67 \%$ mais dor do que os que se julgaram suficientemente condicionados. Mas o possível papel protetor de um bom condicionamento físico não se verifica sempre em populações de trabalhadores, havendo alteração de seu efeito sobre o sistema musculoesquelético em contexto de trabalho físico exaustivo ${ }^{30}$.

Um estudo conduzido entre trabalhadores da indústria de plásticos aponta a interação entre condicionamento e trabalho físico pesado na determinação da dor musculoesquelética ${ }^{30}$ : trabalhadores que relataram mau condicionamento físico apresentaram 3,19 vezes mais chance de dor, em região de pescoço, ombro ou parte alta do dorso, do que aqueles com bom condicionamento, ocorrendo, porém, apenas entre os indivíduos menos expostos fisicamente no trabalho. Os achados evidenciados reiteram o efeito protetor do bom condicionamento físico contra a dor, no entanto, o trabalho com elevada demanda física modificou esse efeito. Entre trabalhadores expostos ao trabalho físico exaustivo, o bom condicionamento físico foi insuficiente para proteger o sistema musculoesquelético. No caso do trabalho físico pesado, trabalhadores bem ou mal condicionados fisicamente apresentaram altas prevalências de dor. Esses dados demonstram que a exposição a altas demandas físicas na ocupação pode ocasionar aumento da morbidade entre trabalhadores, independentemente do condicionamento físico individual ${ }^{30}$. No entanto, esta é uma variável relevante em estudos sobre dor e trabalho e não deve ser negligenciada, pois é necessário entender melhor o seu papel, haja vista o interesse em fatores de risco modificáveis - fatores de estilo de vida, como o condicionamento físico - na perspectiva de prevenção da dor.

A abordagem da dor em MMII, neste estudo, incorporando todos os segmentos na fase analítica baseia-se na discussão acerca da dor múltipla, ou dor concorrente em mais de um segmento corporal $^{40}$. Em estudo com um pool de inquéritos que incorporou a população da limpeza urbana e trabalhadores da indústria de calçados, cerca de $60 \%$ dos indivíduos com dor em tornozelos/pés apresentavam simultaneamente dor nas pernas; apenas $14 \%$ da queixa de dor em tornozelos/pés ocorria isoladamente nesse segmento, ao passo que, em $86 \%$ dos casos, a dor acometia concomitantemente mais de dois segmentos corporais, predominantemente os mais proximais; aqueles com dor nas pernas tinham sete vezes a prevalência de dor em tornozelos/pés daqueles sem dor nas pernas ${ }^{40}$. Desta forma, adotar a análise do desfecho dor em MMII considerando a região corporal, e não os segmentos corporais isoladamente, pode ser o mais adequado e o que se recomenda ${ }^{40}$.

No presente estudo a idade não se associou ao desfecho, ou seja, a prevalência do desfecho é alta, não variando segundo a idade. Uma provável explicação se deve ao fato de a população estudada ser majoritariamente jovem, com pequena variação na amplitude da variável. Assim, em grupo com maior homogeneidade de idade, não houve diferença relevante na ocorrência do desfecho por faixa etária.

A frequência de horas extras é bastante elevada nesta população e chega a ser referida por quase $100 \%$ de motoristas e coletores. Não havendo variabilidade desta exposição, não foi possível verificar diferença na ocorrência do desfecho entre expostos e não expostos que determinasse uma associação razoável ( $\geq 30 \%$ ). No entanto, é possível afirmar que 
as jornadas extenuantes podem ter papel relevante na alta prevalência de dor verificada nesta população de trabalhadores.

Da mesma forma, a alta exposição a posturas anômalas de trabalho é predominante na população estudada, dificultando mostrar o efeito (razão de chances entre expostos e não expostos) desta exposição na ocorrência do desfecho estudado. Assim, ressaltam-se as elevadas prevalências de dor e as altas exposições aqui evidenciadas, haja vista tratar-se de jovens trabalhadores, cujas demandas ocupacionais podem levar a quadros incapacitantes com consequências danosas para suas vidas.

O delineamento transversal, que impossibilita estabelecer a relação temporal de antecedência da exposição ao desfecho, é uma limitação do estudo. Destacam-se como pontos fortes os procedimentos adotados para assegurar privacidade e confidencialidade, aspectos relevantes para a pesquisa no mundo do trabalho e para a validade da pesquisa, particularmente no que se refere a minimizar os vieses de informação e de seleção. Os trabalhadores foram informados sobre a instituição responsável pela pesquisa, uma universidade pública, e sobre a empresa apenas ter permitido o acesso dos pesquisadores aos locais de trabalho, sem ter qualquer participação na pesquisa. Além disso, a coleta de dados por meio de questionário estruturado, adotando uniformidade nos procedimentos quanto às técnicas de entrevista, certamente contribuiu para a validade interna do estudo.

\section{Conclusão}

O trabalho na limpeza urbana expõe os trabalhadores inseridos em atividades de operação e manutenção a altas exigências físicas e psicossociais. Foi possível caracterizar o trabalho quanto a essas demandas, observando algumas variações da exposição entre os grupos ocupacionais. Identificou-se que o trabalho desenvolvido pelos coletores apresenta forte característica física e psicossocial, sendo o grupo com maior percentual de trabalhadores jovens, com baixa escolaridade, raça negra, e que, juntamente com os motoristas, extrapolam comumente a jornada semanal de 44 horas de trabalho. A dor em MMII foi evidenciada entre os TLUs, com $42,1 \%$ de prevalência nos doze meses precedentes e $23,7 \%$ nos últimos sete dias anteriores à coleta de dados. Foram identificados fatores ocupacionais (alta demanda psicológica e física no trabalho, maior tempo de trabalho na empresa) e extraocupacional (condicionamento físico insuficiente) associados à dor em MMII nos TLUs.

O estudo contribui com o conhecimento acerca da dor em MMII entre trabalhadores, evidenciando características do trabalho dos TLUs que poderão ser utilizadas para o planejamento de intervenções voltadas para sua prevenção, como mudanças na organização do trabalho, com adequação do ritmo de trabalho ao tempo disponível, introdução de pausas e recursos para manuseio seguro e confortável de cargas.

\section{Contribuições de autoria}

Lessa RS e Fernandes RCP contribuíram igualmente na concepção do estudo, no levantamento, análise e interpretação dos dados, na elaboração, revisões críticas e na aprovação da versão final publicada e assumem responsabilidade pública integral pelo trabalho realizado e o conteúdo aqui publicado.

\section{Referências}

1. Pereira-de-Paiva $\mathrm{MH}$, Calassa-Albuquerque $\mathrm{MC}$, Latham EE, Furtado-Bezerra C, da-Silva-Sousa A, Cunha-e-Silva-de-Araújo L, et al. Occupational hazards of Brazilian solid waste workers: a systematic literature review. Rev Bras Med Trab. 2017;15(4):364-71.

2. Ziaei M, Choobineh A, Abdoli-Eramaki M, Ghaem H, Jaberi O. Psychological and physical job demands, decision latitude, and work-related social support among Iranian waste collectors. Waste Manag. 2019;95:377-87.

3. Camada IMO, Pataro SMS, Fernandes RCP. Heavy physical work under time pressure: the garbage collection service-a case study. Work. 2012;41(1 Suppl):462-9.
4. Fernandes RCP, Assunção AA, Carvalho FM. Tarefas repetitivas sob pressão temporal: os distúrbios musculoesqueléticos e o trabalho industrial. Cien Saude Colet [Internet]. 2010 [citado em 12 jun 2015];15(3):931-42. Disponível em: http://www.scielo.br/scielo.php?script=sci arttext\&pid =S1413-81232010000300037\&lng=-en

5. Ziaei M, Choobineh A, Abdoli-Eramaki M, Ghaem H. Individual, physical, and organizational risk factors for musculoskeletal disorders among municipality solid waste collectors in Shiraz, Iran. Ind Health. 2018;56(4):308-19.

6. Pataro SMS, Fernandes RCP. Trabalho físico pesado e dor lombar: a realidade na limpeza urbana. Rev Bras Epidemiol [Internet]. 2014 
[citado em 15 jul 2015];17(1):17-30.

Disponível em: http://www.scielo.br/

scielo.php?script $=$ sci_arttext\&pid $=\mathrm{S} 1415$ -

790X2014000100017\&lng $=\mathrm{en}$

7. Cardoso RK, Rombaldi AJ, Silva MC. Distúrbios osteomusculares e fatores associados em coletores de lixo de duas cidades de porte médio do sul do Brasil. Rev Dor [Internet]. 2014 [citado em 14 maio 2015];15(1):13-6. Disponível em: http://www.scielo. br/scielo.php?script $=$ sci_arttext\&pid $=$ S180600132014000100013\&lng $=\mathrm{en}$

8. Abou-Elwafa HS, El-Bestar SF, El-Gilany AH, Awad EES. Musculoskeletal disorders among municipal solid waste collectors in Mansoura, Egypt: a crosssectional study. BMJ Open. 2012;2(5):e001338.

9. Bertoldi CML, Proença RPC. Doença venosa e sua relação com as condições de trabalho no setor de produção de refeições. Rev Nutr [Internet]. 2008 [citado em 27 ago 2018];21(4):447-54. Disponível em: https://www.scielo.br/j/rn/ a/6tf9HRqvsn4wg83wbq3gfJp/abstract/?lang=pt

10. Punnett L. Musculoskeletal disorders and occupational exposures: how should we judge the evidence concerning the causal association? Scand J Public Health. 2014;42(13 Suppl):49-58.

11. D'Souza JC, Franzblau A, Werner RA. Review of epidemiologic studies on occupational factors and lower extremity musculoskeletal and vascular disorders and symptoms. J Occup Rehabil. 2005;15(2):129-65.

12. Coenen P, Parry S, Willenberg L, Shi JW, Romero L, Blackwood DM, et al. Associations of prolonged standing with musculoskeletal symptoms - A systematic review of laboratory studies. Gait Posture. 2017;58:310-8.

13. Messing K, Tissot F, Stock SR. Distal lowerextremity pain and word postures in the Quebec population. Am J Public Health. 2008;98(4):705-13.

14. Ezzat AM, Li LC. Occupational physical loading tasks and knee osteoarthritis: a review of the evidence. Physiother Can. 2014;66(1):91-107.

15. Cardoso JP, Araújo TM, Carvalho FM, Oliveira NF, Reis EJFB. Aspectos psicossociais do trabalho e dor musculoesquelética em professores. Cad Saude Publica [Internet]. 2011 [citado em 12 jun 2018];27(8):1498-506. Disponível em: http://www.scielo.br/scielo.php?script $=$ sci arttext\&pid $=$ S0102-311X2011000800005\&Īng $=$ en

16. Pereira ALP, Fernandes RCP. Trabalho e distúrbios musculoesqueléticos na limpeza urbana. In: Fernandes RCP, Lima MAG, Araújo TM, organizadores. Tópicos em saúde, ambiente e trabalho: um olhar ampliado. Salvador: EDUFBA; 2014. p. $103-28$.

17. Fernandes RCP. Distúrbios musculoesqueléticos e trabalho industrial [tese]. Salvador: Universidade Federal da Bahia; 2004.

18. Pinheiro FA, Tróccoli BT, Carvalho CV. Validação do Questionário Nórdico de Sintomas
Osteomusculares como medida de morbidade. Rev Saude Publica [Internet]. 2002 [citado em 10 mar 2014];36(3):307-12. Disponível em: http://www.scielosp.org/scielo.php?script = sci arttext\&pid $=$ S0034-89102002000300008\&lng $=$ pt

19. Fernandes RCP, Cunha LP, Lima VMC, Santos KOB. Mensurando a demanda física no trabalho: estrutura fatorial e confiabilidade de itens sobre posturas, manuseio de carga e repetitividade. Cad Saude Publica [Internet]. 2019 [citado em 12 mar 2019];35(1):e00123218. Disponível em: http://www.scielo.br/scielo.php?script=sci arttext\&pid $=$ S0102-311X2019000105003\&Īng =en

20. Araújo TM, Karasek R. Validity and reliability of the job content questionnaire in formal and informal jobs in Brazil. SJWEH Suppl. 2008;34(6):52-9.

21. Silvany Neto AM. Bioestatística sem segredos. Salvador: Edição do autor; 2008.

22. Costa EFO, Andrade TM, Silvany Neto AM, Melo EV, Rosa ACA, Alencar MA, et al. Common mental disorders among medical students at Universidade Federal de Sergipe: a cross-sectional study. Braz J Psychiatry [Internet]. 2010 [citado em 22 out 2018];32(1):11-9. Disponível em: http://www.scielo. br/scielo.php?script $=$ sci_arttext\&pid $=$ S151644462010000100005\&lng $=$ en

23. Hahn GJ, Meeker WQ. Assumptions for Statistical Inference. Am Stat. 1993;4(1):1-11.

24. Rothman KJ. Six persistent research misconceptions. J Gen Intern Med. 2014;29(7):1060-4.

25. Greenland S. Randomization, statistics, and causal inference. Epidemiology. 1990;6(1):421-9.

26. Strasak AM, Zaman Q, Pfeiffer KP, Göbel G, Ulmer $H$. Statistical errors in medical research - a review of common pitfalls. Swiss Med Wkly. 2007;137(34):44-9.

27. ElWahab EWA, Eassa SM, Lotfi SE, El Masry SA, Shatat HZ, Kotkat AM. Adverse health problems among municipality workers in Alexandria (Egypt). Int J Prev Med. 2014;5(5):545-56.

28. Brasil. Ministério do Trabalho. Classificação Brasileira de Ocupações. CBO 5142-15: varredor de rua [Internet]. Brasília, DF: Ministério do Trabalho; 2019 [citado em 23 mar 2019]. Disponível em: https://www.ocupacoes.com.br/cbo-mte/514215varredor-de-rua

29. Guérin F, Laville A, Daniellou F, Duraffourg J, Kerguelen A. Compreender o trabalho para transformá-lo: a prática da ergonomia. São Paulo: Blucher; 2001.

30. Mascarenhas ALM, Fernandes RCP. Aptidão física e trabalho físico pesado: como interagem para a ocorrência de distúrbio musculoesquelético? Cad Saude Publica [Internet]. 2014 [citado em 14 out 2016];30(10):2187-98. Disponível em: http://www.scielo.br/scielo.php?script $=$ sci

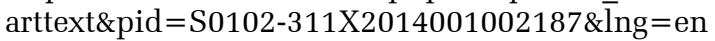


31. Vasconcelos RC, Lima FPA, Abreu ACMS, Silva RCR, Camarotto JA, Murta EP. A estratégia de "redução" e a carga de trabalho dos coletores de lixo domiciliar de uma grande cidade: estudo de caso baseado na Análise Ergonômica do Trabalho. Rev Bras Saude Ocup [Internet]. 2008 [citado em 18 out 2016];33(117):50-9. Disponível em: http://www.scielo.br/scielo.php?script =sci arttext\&pid $=$ S0303-76572008000100006\&lng $=$ en

32. Dul J, Weerdmeester B. Postura e movimento. In: Dul J, Weerdmeester B. Ergonomia prática. 3a ed. Itiro Lida, tradutor. São Paulo: Blucher; 2012. p. 17-54.

33. Brasil. Ministério da Saúde. Secretaria de Vigilância em Saúde. Departamento de Vigilância em Saúde Ambiental e Saúde do Trabalhador. Dor relacionada ao trabalho: lesões por esforços repetitivos (LER): distúrbios osteomusculares relacionados ao trabalho (Dort). Brasília, DF: Ministério da Saúde; 2012.

34. Barros N Jr. Insuficiência venosa crônica. In: Pitta GBB, Castro AA, Burihan E, editores. Angiologia e cirurgia vascular: guia ilustrado. Maceió: UNCISAL; 2003. p. 2-17.
35. Chester MR, Rys MJ, Konz SA. Leg swelling, comfort and fatigue when sitting, standing, and sit/ standing. Int J Ind Ergon. 2002;29(5):289-96.

36. Lin YH, Chen CY, Cho MH. Influence of shoe/ floor conditions on lower leg circumference and subjective discomfort during prolonged standing. Appl Ergon. 2012;43(5):965-70.

37. Messing K, Stock S, Tissot F. The importance of not standing too long. Arch Environ Occup Health. 2013;68(2):128-9.

38. Huang GD, Feuerstein M, Sauter SL. Occupational stress and work-related upper extremity disorders: concepts and models. Am J Ind Med. 2002;41(5):298-314.

39. Lanfranchi JB, Duveau A. Explicative models of musculoskeletal disorders (MSD): from biomechanical and psychosocial factors to clinical analysis of ergonomics. Eur Rev Appl Psychol. 2008;58(4):201-13.

40. Fernandes RCP, Pataro SMS, Carvalho RB, Burdorf A. The concurrence of musculoskeletal pain and associated work-related factors: a cross sectional study. BMC Public Health [Internet]. 2016 [citado em 10 ago 2019];16:628. Disponível em: https://bmcpublichealth.biomedcentral.com/ articles/10.1186/s12889-016-3306-4 